\title{
Rins de Baleia Minke (Baleanoptera acutorostrata): arquitetura e estrutura ${ }^{1}$
}

\author{
Carlos A.P. Sarmento ${ }^{2}$, Amanda O. Ferreira², Elaine A.F. Rodrigues ${ }^{2}$, Giuliano G. \\ Lesnau$^{2}$, Rose Eli G. Rici ${ }^{2}$, Dilayla K. Abreu², Caio Biasi ${ }^{2}$ e M. Angelica Miglino ${ }^{2}$
}

\begin{abstract}
Sarmento C.A.P., Ferreira A.O., Rodrigues E.A.F., Lesnau G.G., Rici R.E.G., Abreu D.K., Biasi C. \& Miglino M.A. 2012. [Kidney of Minke Whale (Baleanoptera acutorostrata): Architecture and structure.] Rins de Baleia Minke (Baleanoptera acutorostrata): arquitetura e estrutura. Pesquisa Veterinária Brasileira 32(8):807-811. Departamento de Cirurgia, Setor de Anatomia dos Animais Domésticos e Silvestres, Universidade de São Paulo, Av. Prof. Dr. Orlando Marques de Paiva 87, São Paulo, SP 05508-270, Brazil. E-mail: sarmento@usp.br

Among marine mammals, whale is one of the most attention-arousing animals, especially concerning its urinary tract. This system follows the pattern of mammals with regard to its constitution, however, it differs in renal morphology and number of lobes, which, in turn, form complete reniculi, agglutinated in hundreds. This structure is supported by fibrous connective tissue, but highly capable of maintaining electrolyte balance. Six pairs of kidneys of Minke whale (Balaenoptera acutorostrata), collected in 1982, in Cabedelo, Paraiba, Brazil, in the last fishing allowed, were dissected. These kidneys were preserved in $10 \%$ formaldehyde and they presented a very large histologic layer of collagen surrounding the medullary wall. The urinary collecting duct form papillary glasses, that reach a single collecting center which discharges in the ureter. It was found that the kidney of Minke whale has a lobe characteristic, with, on average, 700 reniculi; each reniculus has anatomical and functional characteristics of a unipyramidal kidney, with an inner layer (medulla), and an outer layer (cortex), and independent irrigation, with formation of individually arcuate arteries, as observed in unipyramidal terrestrial mammals. However, the set gathering all these reniculi constitutes, in the end, a multilobular and polipyramidal kidney, contrary to the morphology of most terrestrial mammals. It was not possible to distinguish the renicular cortex structures of the Minke whale in the level of light microscopy. Through scanning electron microscopy, it was possible to visualize a cortical layer located between two fibrous capsules. This joint, in turn, consists of connective tissue, which, along with a layer of collagen and elastic fibers, separates the cortex from the medulla; the kidney glomeruli were visualized, completely taken by the glomerular vessels and arranged into several layers. One notices that the glomerular cavity is almost a virtual space into which the glomerular filtrate is drained, and it does not present a globular shape. Vascularization is increased in the medullary region. The difference between the kidneys of terrestrial and marine mammals consists in the arrangement of morphological components, favoring the organ's physiology.
\end{abstract}

INDEX TERMS: Whale, Baleanoptera acutorostrata, kidney, mammals.

RESUMO.- Entre os mamíferos marinhos, a baleia é um dos animais que mais desperta atenção, especialmente no atinente ao seu sistema urinário. Este sistema segue o

\footnotetext{
${ }^{1}$ Recebido em 25 de janeiro de 2012.

Aceito para publicação em 17 de maio de 2012

${ }^{2}$ Departamento de Cirurgia, Setor de Anatomia dos Animais Domésticos e Silvestres, Universidade de São Paulo (USP), Av. Prof. Dr. Orlando Marques de Paiva 87, São Paulo, SP 05508-270, Brasil. *Autor para correspondência: sarmento@usp.br
}

padrão entre os mamíferos quanto a sua constituição, entretanto, difere na morfologia renal, em número de lobos, que por sua vez, forma renículos completos, aglutinados às centenas. Esta estrutura é sustentada por tecido conjuntivo fibroso, mas altamente capaz de manter o equilíbrio hidroeletrolítico. Foram dissecados 6 pares de rins de baleia Minke (Balaenoptera acutorostrata), colhidos em 1982, Cabedelo, Estado da Paraíba, Brasil, na última pesca autorizada. Estes rins estavam conservados em formol 10\% e 
apresentaram uma camada histológica de colágeno muito grande circundando a parede medular. 0 duto coletor urinário forma cálices papilares, desembocando num único centro coletor que desemboca no ureter. Verificou-se que o rim da baleia Minke apresenta característica lobulada possuindo em média 700 renículos, cada renículo possui características anatômicas e funcionais de um rim unipiramidal, com uma camada interna (medula), e uma camada externa (córtex), e irrigação independente, com formação das artérias arqueadas individualmente, como observadas em mamíferos terrestres unipiramidais. Entretanto, o conjunto destes renículos constitui ao final um rim multilobular e polipiramidal, contrariando a morfologia da maioria dos mamíferos terrestres. Não foi possível distinguir ao nível de microscopia de luz as estruturas do córtex renicular da baleia Minke. Na microscopia eletrônica de varredura foi possível visualizar uma camada cortical que fica localizada entre duas cápsulas fibrosas. Esta junção por sua vez é feita por tecido conjuntivo o qual juntamente com uma camada de colágeno e fibras elásticas, separa o córtex da medula , foram visualizados os glomérulos renais, completamente tomados pelos vasos glomerulares e dispostos em várias camadas. Percebe-se que a cavidade glomerular é praticamente um espaço virtual para onde o filtrado glomerular é drenado, não apresentando o formato globular. A vascularização intensifica-se ao chegar à região medular. A diferença entre rins de mamíferos terrestres e marinhos está na disposição dos componentes morfológicos, favorecendo a fisiologia do órgão.

TERMOS INDEXADOS: Baleia, Baleanoptera acutorostrata, rim, mamíferos.

\section{INTRODUÇÃO}

A ordem Cetacea é composta por mamíferos exclusivamente aquáticos, que possuem sangue quente e respiração pulmonar, vindo à superfície em intervalos regulares para realizar a respiração (Reis 2000). Os cetáceos, tais como as baleias, os botos e os golfinhos, habitam todos os oceanos e mares, porem poucos habitam rios. É importante ressaltar que esses animais sempre despertaram o interesse e a imaginação das pessoas, devido a sua inteligência, amabilidade e histórias contadas a seu respeito (Hetzel \& Lodi 1993).

O sistema urinário dos cetáceos é composto por rins ovais, alongados e multilobados, que produzem urina altamente concentrada, reduzindo assim a perda de água (Geraci 1986, Geraci \& Lounsbury 1993a, 2005a). Os rins contêm unidades individuais conhecidas como renículos que podem variar de centenas a milhares, sendo agrupados por uma fina cápsula de tecido conjuntivo fibroso. 0 número de renículos na ordem Cetácea varia entre 75 a 170 nos golfinhos e 600 a 700 nas baleias (Ommanney 1932, Gihr \& Kraus 1970).

A alta capacidade de manter o balanço hídrico e homeostase eletrolítica durante longos períodos de tempo, com total abstinência de comida e água requer mecanismos ainda mais específicos projetados para manter a homeostase interna. Ocupando extremos de salinidade do ambiente, requer a adaptação a ambos, bem como períodos prolongados de jejum, fornecem uma indicação do âmbito da aplicação dinâmica osmorregulatória altamente capacitada dos mamíferos marinhos, tornando os mesmos um modelo interessante para o estudo da função renal por mais de um século (Ortiz 2001).

Os renículos podem estar compostos por uma papila renal (renículo simples) ou por duas ou mais papilas renais (renículo composto). Algumas vezes estas papilas podem estar unidas umas as outras, e neste caso o córtex e a medula apresentam-se fundidos . Cada renículo, de aproximadamente $1 \mathrm{~cm}$ de diâmetro, é funcional e autônomo, possuindo sua própria medula, córtex e papila. Normalmente uma camada de colágeno, fibras elásticas e musculatura lisa são observadas na junção cortico-medular circundando a pirâmide medular, sendo esta característica evidenciada unicamente nos cetáceos (Hedges et al. 1979)

Os glomérulos são estruturas de tamanho homogêneo. Vários renículos compartilham um mesmo ducto urinário que conduz ao ureter correspondente. Os rins desempenham papel de suma importância na manutenção do equlíbrio hídrico, eletrolítico e ácido-básico, além de participar da síntese de hormônios como a eritropoetina, que é imprescindível para a maturação dos eritrócitos. Os néfrons exercem as funções de manter a homeostase, e promover a depuração do sangue mediante filtração glomerular e reabsorção e secreção tubular (Osborne \& Finco 2005).

0 objetivo desta investigação é realizar a descrição da arquitetura e da estrutura renal da Baleia Minke (Baleanoptera acutorostrata), visando estabelecer comparação com outros cetáceos e entender a fisiologia renal desses mamíferos aquáticos.

\section{MATERIAL E METODOS}

Os seis (6) pares de rins da baleia Minke (Balaenoptera acutorostrata) conservados em formol $10 \%$, pertencem ao acervo da Anatomia dos Animais Domesticos e Silvestres do Departamento de Cirurgia da Faculdade de Medicina Veterinária e Zootecnia da USP.

No processamento das amostras para Microscopia de Luz (Paraplast Embedding Media - Paraplast Plus, Oxford Lab., USA), o material foi desidratado em série crescente de concentração de alcoóis (70-100\%) e diafanizado em xilol, com posterior inclusão em paraplast. Nos blocos de paraplast foram feitos cortes de $5 \mu \mathrm{m}$ de espessura, utilizando o micrótomo (Leika, Alemanha). Em seguida, os cortes foram colocados sob lâminas e corados com $\mathrm{HE}$, e fotodocumentados no microscopio Nikon Eclipse E800.

No processamento para microscopia eletrônica de varredura o material coletado foi lavado em água destilada e pós-fixado em solução de tetróxido de Ósmio a 1\% em PBS 0,1\%. Posteriormente as amostras foram lavadas em solução PBS 0,10\%, e em água destilada. E ao final, o material foi desidratado em séries crescentes de alcoóis em concentrações de $50 \%$ a $100 \%$. As amostras foram secas em aparelho de ponto crítico Balzers CPD 020 (FMVZ-USP). Após, os espécimes foram montados em bases metálicas de alumínio (stub) apropriadas para a microscopia eletrônica de varredura, utilizando-se cola de carbono. Na sequência, foram submetidas a um revestimento metálico (sputting) com ouro no aparelho metalizador EMITECH K550 (FMVZ-USP), sendo analisadas e fotografadas em microscópio eletrônico de varredura LEO 435VP (FMVZ-USP). 


\section{RESULTADOS E DISCUSSÃO}

Os rins de baleia apresentam uma camada de tecido adiposo ao seu redor, constituindo a gordura perirenal. Afastando a gordura perirenal, encontra-se a cápsula renal, constituída de tecido fibroso, mas diferentemente dos mamíferos terrestres, esta cápsula está fixada à borda de cada renículo, por meio de tecido conjuntivo. Esta característica configura o isolamento morfológico completo de cada renículo, ao contrário dos rins lobados de mamíferos terrestres citados pelos tratadistas (Fig.1A). Estes rins apresentam coloração acastanhada (material formolizado) ou avermelhada intensa (material não formolizado) devido à presença de sangue, com um hilo renal, por onde adentram artérias renais e inervação e saem veias renais, vasos linfáticos e ureteres. A diferença se faz na presença de uma veia adentrando a extremidade caudal de cada rim fazendo comunicação interna com a veia renal, dentro do parênquima renal, Este vaso em comunicação constitui a veia porta renal.

A partir do material analisado, verificou-se que o rim da baleia Minke apresentou comprimento médio de 58,3 $\mathrm{cm}$.,característica lobulada possuindo em média 700 renículos (Fig.1A), cada renículo possuía características anatômicas e funcionais de um rim, com medula e córtex renal, e irrigação independente, como é encontrada em mamíferos terrestres. Entretanto, os mesmos apresentaram formato multilobular e polipiramidal ao contrário da maioria dos mamíferos terrestres que apresenta os rins não lobulados e unipiramidais (Walter \& Sayles 1949, Beauch 2002). Esta característica torna os rins dos cetáceos uma estrutura bastante especializada (Ommaney1932, Ortiz 2001). No entanto, segundo Bester 1975 o rim reniculado, não possui a habilidade de concentrar urina e eletrólitos compatíveis com as excelentes características anatômicas que possui. Não foi possível distinguir ao nível de microscopia de luz as estruturas do córtex renicular no rim de baleia Minke. Esse achado corrobora com o encontrado por (Pfeiffer 1997) que realizou um estudo semelhante a este em baleia Beluga.

À microscopia óptica, é possível notar a distribuição homogênea e simétrica dos glomérulos às margens dos vasos sanguíneos (Fig.1B-D). 0 tecido conjuntivo de colágeno é uma constante, entretanto, não tão presente quanto a presença de arteríolas eferentes promovendo a reabsorção da água ao longo dos néfrons (Fig.1C), conferindo assim a coloração intensa dos rins. Os vasos sanguíneos que distribuem o sangue entre os glomérulos (arteríola aferente) apresentam-se em estrutura unicelular formando o capilar (Fig.1C).
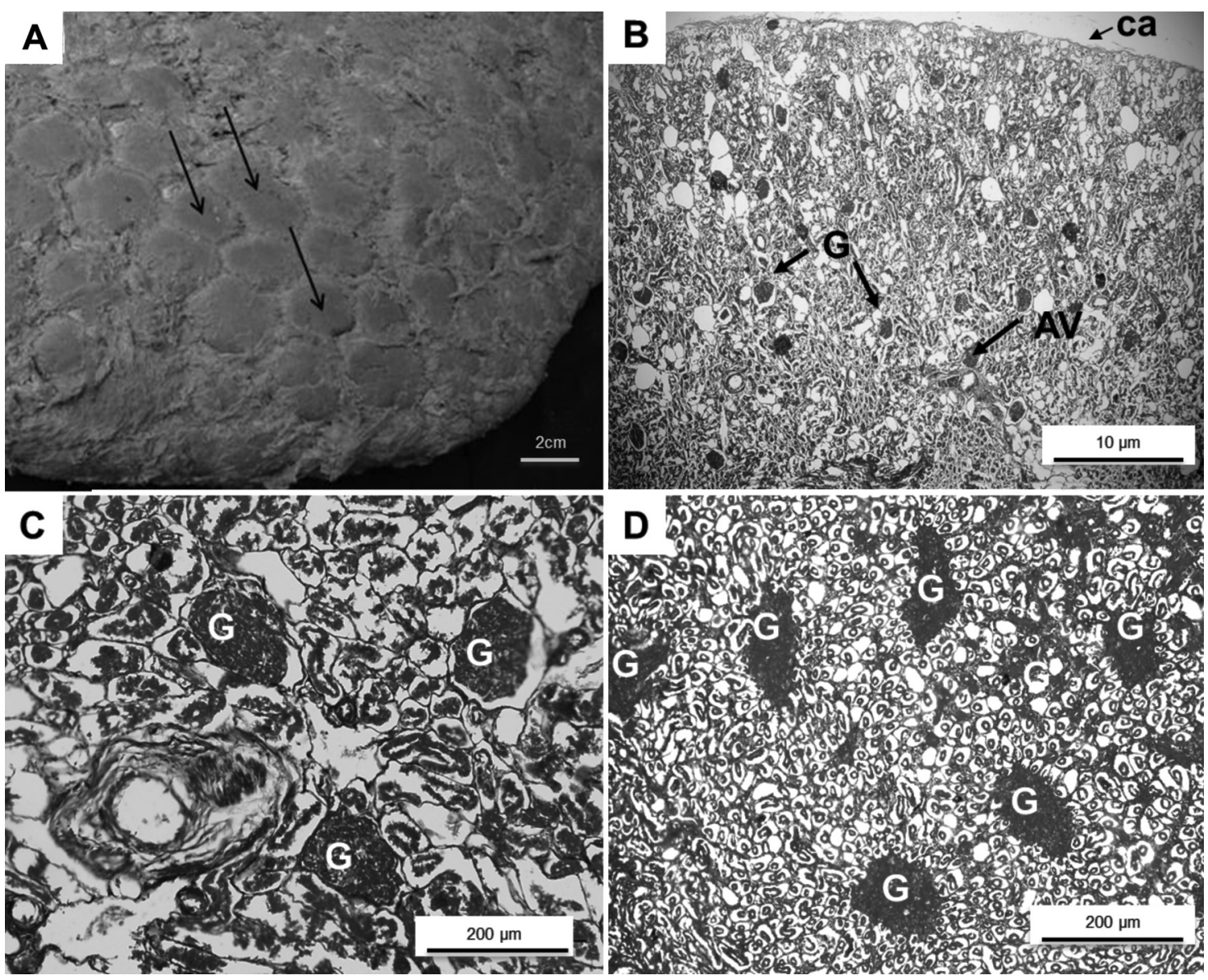

Fig.1. (A-D) Em A, fotomacrografia de rim da baleia Minke, onde são observados pelas setas os renículos do rim. Na figura B, fotomicrografia da vista panorâmica da camada cortical do rim mostrando a cápsula (ca), glomérulos (G) e artérias e veias interlobulares (AV). Nas figuras C e D, fotomicrografia da camada cortical do rim evidenciando os glomérulos (G). Tricômio de Masson. Em A, barra com $2 \mathrm{~cm}$. Em B, barra com 10 um. Em C e D, barra com 200 um. 
Mediante microscopia eletrônica de varredura foi possível visualizar uma camada cortical que fica localizada entre duas cápsulas fibrosas. Esta junção por sua vez é feita por tecido conjuntivo o qual juntamente com uma camada de colágeno e fibras elásticas, separa o córtex da medula (Fig.2B). Segundo Henc e colaboradores 1986, esta é uma característica exclusiva dos cetáceos.

A análise histológica demonstra uma alta concentração de vasos sanguíneos localizados na medula renal os quais tem como objetivo principal reduzir o mecanismo de troca contracorrente nesse sitio. Cada glomérulo apresentou pelo menos duas camadas de vasos ao seu redor, ou seja, entre um glomérulo e outro, há pelo menos quatro camadas de vasos sanguíneos separando-os (Fig.1D). Camadas estas oriundas do enovelamento vascular ao redor do néfron, formando um intenso plexo para maximizar as trocas e absorção de água (Fig.1C e Fig.2B).Esse fato aliado a complexa estrutura dos renículos, parece ser uma adaptação dos cetáceos ao ambiente marinho (Pfeiffer 1997). A análise realizada pela microscopia eletrônica de varredura permite a visualização dos glomérulos renais, completamente tomados pelos vasos glomerulares e dispostos em várias camadas. Percebe- -se que a cavidade glomerular é praticamente um espaço virtual para onde o filtrado glomerular é drenado (Fig.2B). Desta forma, o glomérulo não apresenta necessariamente o formato globular, e apresenta-se implantado em uma cavidade com liberdade de distensão no caso de hipertensão (Fig.2C).

Foi constantemente notada a presença de artérias arqueadas ao redor ou próximo ao glomérulo (Fig.2D). Os capilares glomerulares formam uma intensa rede vascular, para ampliar sua área de contato (Fig.2D) e maximizar a filtragem glomerular.

Observou-se que na camada cortical, os vasos se direcionam para o córtex medular, onde a vascularização vai se intensificando. 0 contingente de glomérulos é exclusivo do córtex renicular (Fig.2A e Fig.2B). Não foi possível identificar demais estruturas como células do parênquima, devido ao grande tempo de conservação deste material.

Após nosso estudo, podemos inferir que a diferença na estrutura renal entre mamíferos aquáticos e terrestres, se deve ao fato dos rins possuírem estruturas especializadas, as quais estão relacionadas à sua fisiologia quanto à regulação de água e eletrólitos, e ao ambiente com alto grau de salinidade que estes animais vivem.
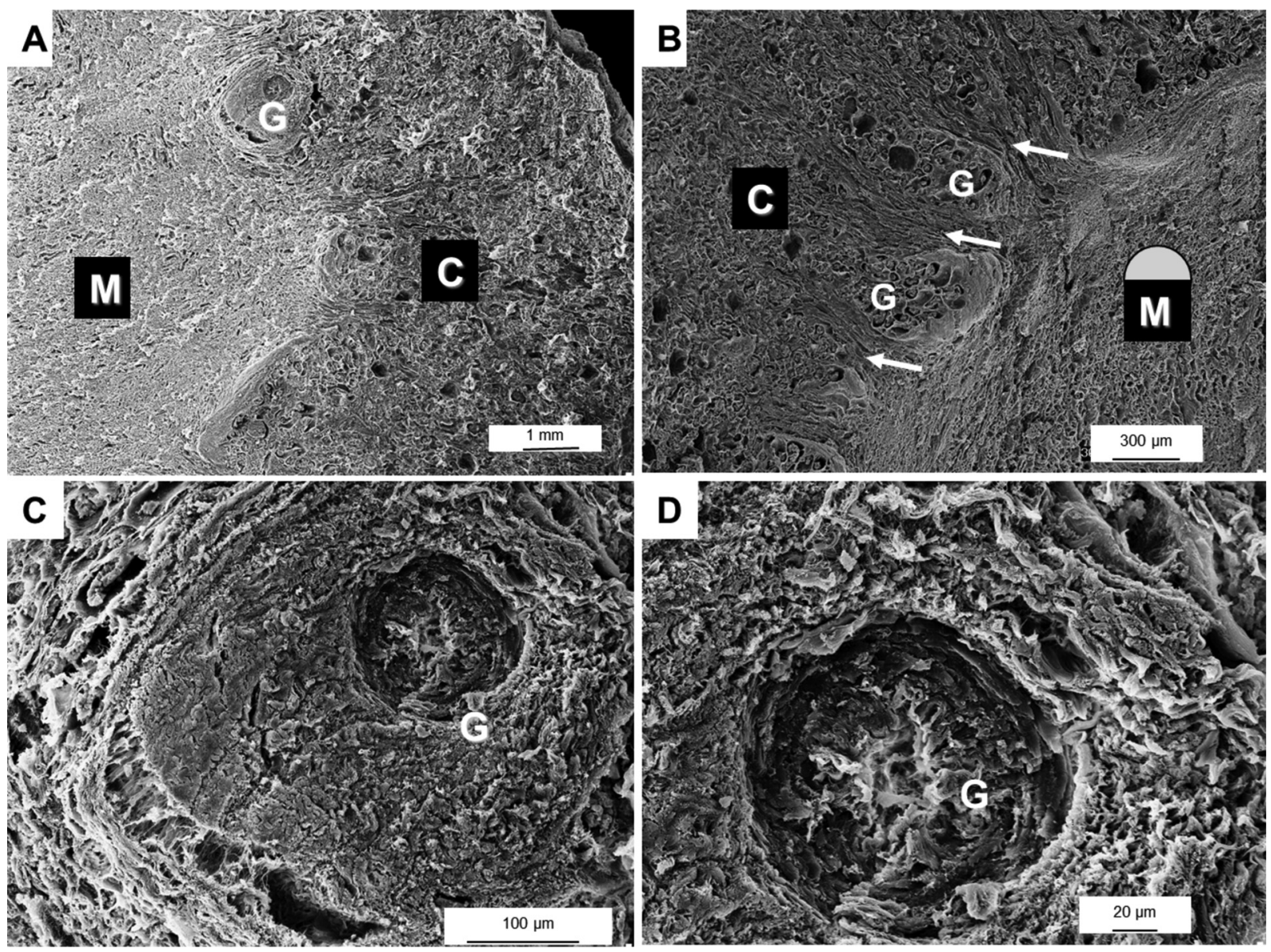

Fig. 2. (A-D) Em A, eletromicrografia de varredura mostrando a camada cortical (C) e camada medular (m), onde é possível identificar o glomérulo (G). Em B, micrografia eletrônica de varredura onde é possível evidenciar glomérulos(G) (corpúsculos renais), camada medular (M) e camada cortical (C). Na seta pode-se observar os raios medulares formados por fibras elásticas que separam as camadas. Artérias Arqueadas (AA) em D. Em C e D, micrografia eletrônica de varredura identificando o glomérulo (G). Em A, barra com 1 mm. Em B, barra com 300 um. Em C, barra com 100 um. Em D, barra com 20 um. 


\section{REFERÊNCIAS}

Baker J.R. 1992. Causes of mortality and parasites and incidental lesions in dolphins and whales from British waters. Vet. Rec. 130:569-572.

Bester M.N. 1975. The functional morphology of the kidney of the Cape fur seal, Arctocephalus pusillus (Schreber). Modoqua Ser. II 4:69-92.

Beuchat C.A. 2002. Kidney, structure and function, p.646-649. In: Perrin W. F., Wursig B., Thewissen J.G.M. (Eds), Encyclopedia of Marine Mammals. Academic Press, San Diego.

Bratton G., Spainhour C., Flory W., Reed M. \& Jayko K. 1993. Presence and potential effects of contaminants, p.701-744. In: Burns J.J., Montague J.J., Cowles C.J. \& Lawrence K.S. (Eds), The Bowhead Whale. Society for Marine Mammalogy, Lawrence, KA, USA

Byrne C., Balasubramanian R., Overton E. \& Albert T. 1985. Concentrations of trace metals in the bowhead whale. Marine Pollution Bull. 14:497-498.

Di Guardo G., Agrimi U., Morelli L., Cardeti G., Terracciano G. \& Kennedy S. 1995. Postmortem investigations on cetaceans found stranded on the coast of Italy between 1990 and 1993. Vet. Rec. 136:439-442.

Domingo M., Visa J., Pumarola M., Marco A., Ferrer L., Rabanal R. \& Kennedy S. 1992. Pathologic and immunocytochemical studies of distemper in striped dolphins (Stenella coeruleoalba). Vet. Pathol. 29:1-10.

Hetzel B. \& Lodi L. 1994. Baleias, Botos e Golfinhos. Editora Nova Fronteira, Rio de Janeiro.

McFall J., Antoine S. \& Overton E. 1986. Organochlorine compounds and polynuclear aromatic hydrocarbons in tissues of subsistence harvested bowhead whales, Balaena mysticetus. Report to the North Slope Borough, Barrow, Alaska, From the Center for Bio-Organic Studies. Univ. of New Orleans, New Orleans, L.A.

O’Hara T.M., Krahn M.M., Boyd D., Becker P.R. \& Philo L.M. 1999. Organochlorine contaminant levels in Eskimo harvested bowhead whales of arctic Alaska. J. Wildl. Dis. 35:741-752.

Ommanney F.C. 1932. The urino-genital system of the fin whale (Balaenoptera physalus). With appendix: The dimensions and growth of the kidney of blue and fin whales. Discovery Rep. 5:363-466.

Overton E., Byrne C., McFall J., Antoine S. \& Laseter J. 1983. Preliminary observations on tissue pollutant levels in subsistence harvested Bowhead Whales (Balaena mysticetus). Submitted as paper SC/35/PS17 to the International Whaling Commission Scientific Committee, International Whaling Commission, Cambridge, United Kingdon.

Pfeiffer C.J. 1997. Renal cellular and tissue specializations in the bottlenose dolphin (Tursiops truncatus) and beluga whale (Delphinapterus leucas). Aquatic Mammals 23(2):75-84.

Rudy M.O. 2001. Osmoregulation in marine mammals. J. Exp. Biology 204: 1831-1844.

Sweeney J.C. \& Ridgway S.H. 1975.Common diseases of small cetaceans. J. Am. Vet. Med. Assoc. 167:533-539.

Walter H.E. \& Sayles L.P. 1949. Biology of the Vertebrates. MacMillan Company, New York. 